0749-6419(95)00025-9

\title{
FORMATION OF SHEAR BANDS IN PLANE SHEET
}

\author{
Guo-Chen Li and Chen ZHU
}

Institute of Mechanics, Academia Sinica

\begin{abstract}
Alstract - The formation of shear bands in plane sheet is studied, both analytically and experimentally, to enhance the fundamental understanding of this phenomenon and to develop a capability for predicting material failure. The evolution of voids is measured and its interaction with the process of shear banding is examined. The evolving dilatancy in plasticity is shown to have a vital role in analysing the shear-band type of bifurcation, and tremendously reduces the theoretical value of critical stresses. The analyses, referring to both localized and diffuse modes of bifurcation, fairly explain the corresponding observations obtained through testing a dual-phase steel sheet and provide a justification of the constitutive model used.
\end{abstract}

\section{INTRODUCTION}

A salient feature of incipient ductile damage is the formation of shear bands, which are commonly categorized as material bifurcation. Shear bands act as both a precursor to fracture and a mechanism for localization of large plastic strain. Developing the ability to predict material failure in this aspect is of great importance for the efficient control of the metal-forming process.

HIL,L and HuTCHINSON [1975] formed an original and theoretical framework using incompressible plasticity theory to analyse the localized mode of bifurcation into a shear-band pattern. However, ANAND and SPITZIG [1980] indicated that employing the conventional theories of plasticity to predict the initiation of shear bands resulted in unacceptable critical strains, which were too high to be comparable with their experiments of aged maraging steel.

YамAMOTO [1978] applied Gurson's model (GURSON [1977]) to constitutive formulation for shear-band analysis and concluded that low strain-hardening accompanied by a certain amount of initial void volume fraction or localization with an initial imperfection could have critical strains much reduced to a reasonably finite strain level. OHNO and Hutchinson [1984] also showed that flow localization in voided solids was highly sensitive to non-uniformity in void distribution. The effect of yield surface curvature on plastic flow localization was studied by TVERGAARD [1987] and led to another approach to relax the overly stiff response. Strain softening, no matter how small, is well known (BAZANT [1988]; LI [1988, 1990, 1991]) as an effect which much promotes localization and has recently drawn an expanding awareness of its importance. But, as pointed out by BAZANT [1988], a local constitutive theory inevitably leads to a softening zone of negligible thickness. To obtain relief from this dilemma, gradient plasticity modelling in a non-local sense has been tried by SCHREYER [1990] and Murlhaus and AIfantis [1991]. They proposed that the stress at a point 
was dependent on the gradient of plastic strain or the average strain within some symmetric neighbourhood of that point. Aiming at the prediction for the onset of shear banding, LEE [1992] derived a necessary and sufficient condition for hyperbolicity in field equations, using Eulerian formulation for finite elastoplastic deformation of compressible materials.

In this paper, the formation of shear bands in plane sheet is studied both analytically and experimentally. The evolution of voids is measured and its interaction with the process of shear banding is examined. The evolving dilatancy in plasticity, or say, the rate of porosity (instead of claiming the amount of void volume fraction itself), is shown acting a vital role in shear-band type of bifurcation. Both the localized mode, following that given by HILL and HuTCHINSON [1975], and a diffuse mode are analysed for theoretical prediction. Taking into account the current plastic dilatation caused by internal voiding, we can obtain reasonable values of critical stress and strain under practical porosity.

\section{THEORETICAL FORMULATIONS}

\section{II.I. Variational statements on bifurcation analysis}

According to the variational formulation of HiLL [1959], any incremental solution of the boundary-value problem, unique or not, can be characterized by a functional namely that

$$
\mathrm{II}=\int_{v} E\left(V_{j, i}\right) \mathrm{d} v-\int_{s_{f}} \dot{F}_{i} V_{i} \mathrm{~d} s
$$

is stationary. For the sake of simplicity, here and hereafter, the body force rate is neglected. In eqn (1), $V_{j}$ is the velocity vector; a comma before the subscript $i$ denotes the partial derivative with respect to the Cartesian coordinate $x_{i} . E\left(V_{j, i}\right)$ is a homogeneous function of degree two in the velocity gradients. By the device of choosing a reference state that is instantaneously coincident with the current state under consideration, $s$ and $v$ are then, respectively, the surface and volume at the time specified in an updated Lagrangian system; $s_{f}$ is a segment of surface $s$ over which traction rates $\dot{F}_{i}$ are prescribed.

If the nominal stress rates $\dot{T}_{i j}$ satisfy

$$
\dot{T}_{i j}=\partial E / \partial\left(V_{j, i}\right)
$$

then the formulation of rate equilibrium at arbitrary amounts of deformation can be given by the virtual work equation; that is

$$
\int_{v} \delta E \mathrm{~d} v=\int_{v} \dot{T}_{i j} \delta V_{j, i} \mathrm{~d} v=\int_{s_{f}} \dot{F}_{i} \delta V_{i} \mathrm{~d} s
$$

Equivalently, we can say that the first variation of eqn (1) vanishes; that is

$$
\delta \mathrm{II}=\int_{v} \dot{T}_{i j} \delta V_{j, i} \mathrm{~d} v-\int_{s_{f}} \dot{F}_{i} \delta V_{i} \mathrm{~d} s=0 .
$$


In terms of the Jaumann rate $\mathcal{D} / \mathcal{D} t$ of Kirchhoff stress $\tau_{i j}$, the nominal stress rates $\dot{T}_{i j}$ can be expressed as

$$
\dot{T}_{i j}=\frac{\mathcal{D} \tau_{i j}}{\mathcal{D} t}-\sigma_{i k} D_{j k}-\sigma_{j k} D_{i k}+\sigma_{i k} V_{j, k}
$$

where $\sigma_{i k}$ the Cauchy stress and the rate of deformation $D_{i k}$ is known to be related to the velocity components $V_{i}$ by

$$
D_{i k}=\frac{1}{2}\left(V_{i, k}+V_{k, i}\right)
$$

The constitutive equation takes the form of

$$
\frac{\mathcal{D} \tau_{i j}}{\mathcal{D} t}=L_{i j k l} D_{k l}
$$

with the elastic-plastic stiffness tensor

$$
L_{i j k l}=L_{j i k l}=L_{k l i j}=L_{k l i j} .
$$

Thus the function $E\left(V_{j, i}\right)$ can be written as (see HILL [1967])

$$
\begin{aligned}
E\left(V_{j, i}\right) & =\frac{1}{2}\left[\frac{\mathcal{D} \tau_{i j}}{\mathcal{D} t} D_{i j}-\sigma_{i j}\left(2 D_{i k} D_{j k}-V_{k, i} V_{k, j}\right)\right] \\
& =\frac{1}{2} \dot{T}_{i j} V_{j, i}
\end{aligned}
$$

and the relationship between $E\left(V_{j, i}\right)$ and $\dot{T}_{i j}$ satisfies the requirement of eqn (2). Substituting eqn (5) into eqn (3) or making use of eqn (8a) we can obtain the variational formulation given by MCMEEKING and RICE [1975] for problems of large elasticplastic deformation. In the absence of body force rate, it yields

$$
\int_{v}\left[\frac{\mathcal{D} \tau_{i j}}{\mathcal{D} t} \delta D_{i j}-\frac{1}{2} \sigma_{i j} \delta\left(2 D_{i k} D_{j k}-V_{k, i} V_{k, j}\right)\right] \mathrm{d} v=\int_{s_{f}} \dot{F}_{i} \delta V_{i} \mathrm{~d} s
$$

BURKE and NIX [1979] applied the theory of HiLL [1959,1967] to bifurcation analysis. Under the condition of dead (conservative) loading and for linear comparison solids, they concluded that the eigenvalue problem of bifurcation can be expressed by the variational equality

$$
\delta Q=0
$$

and

$$
Q=\frac{1}{2} \int_{v}\left[\frac{\mathcal{D} \bar{\tau}_{i j}}{\mathcal{D} t} \bar{D}_{i j}-\sigma_{i j}\left(2 \bar{D}_{i k} \bar{D}_{j k}-\bar{V}_{k, i} \bar{V}_{k, j}\right)\right] \mathrm{d} v
$$

where a superposed bar is used to denote the difference between corresponding quantities. 
Owing to the arbitrary nature and the infinitesimal virtue of the barred quantities, we may prefer to substitute the bar by the symbol $\delta$ and to replace the $\delta$ in eqn (10a) by $\delta^{*}$. Hereafter, we use $\delta V_{i}$, instead of $\bar{V}_{i}$, to represent any possible pattern of velocity being bifurcated and take $\delta^{*}\left(\delta V_{i}\right)$ as the variation between different patterns. So that

$$
Q=\frac{1}{2} \int_{v}\left[\frac{\mathcal{D} \delta \tau_{i j}}{\mathcal{D} t} \delta D_{i j}-\sigma_{i j}\left(2 \delta D_{i k} \delta D_{j k}-\delta V_{k, i} \delta V_{k, j}\right)\right] \mathrm{d} v
$$

where $\delta D_{i j}$ is related to $\delta V_{i}$ and $\mathcal{D} \delta \tau_{i j} / \mathcal{D} t$ through eqns(6) and (7), respectively. It is not difficult to justify that, when the variation of the external force rate can be neglected, or say, under dead loading, we should have

$$
Q=\frac{1}{2} \delta^{2} \mathrm{II}
$$

and eqn (10a) rewritten by

$$
\delta^{*} Q=0
$$

The stationary condition given by eqn (11c) for bifurcation analysis can be expressed in the developed form as

$$
\begin{aligned}
\delta^{*} Q & =\int_{v}\left[\frac{\mathcal{D} \delta \tau i j}{\mathcal{D} t}-\sigma_{i k} \delta D_{j k}-\sigma_{j k} \delta D_{i k}+\sigma_{i k} \delta V_{j, k}\right] \delta^{*}\left(\delta V_{j, i}\right) \mathrm{d} v \\
& =\int_{v} \delta \dot{T}_{i j} \delta^{*}\left(\delta V_{j, i}\right) \mathrm{d} v=0
\end{aligned}
$$

Using the divergence theorem, we further have

$$
\begin{aligned}
\delta^{*} Q= & -\int_{V}\left[\left(\frac{\mathcal{D} \delta \tau_{i j}}{\mathcal{D} t}-\sigma_{i k} \delta D_{j k}-\sigma_{j k} \delta D_{i k}+\sigma_{i k} \delta V_{j, k}\right), i\right] \delta^{*}\left(\delta V_{j}\right) \mathrm{d} v \\
& +\int_{s_{f}}\left[\left(\frac{\mathcal{D} \delta \tau_{i j}}{\mathcal{D} t}-\sigma_{i k} \delta D_{j k}-\sigma_{j k} \delta D_{i k}+\sigma_{i k} \delta V_{j, k}\right) \nu_{i}\right] \delta^{*}\left(\delta V_{j}\right) \mathrm{d} s \\
= & -\int_{v} \delta \dot{T}_{i j, i} \delta^{*}\left(\delta V_{j}\right) \mathrm{d} v+\int_{s_{f}} \delta \dot{T}_{i j} \nu_{i} \delta^{*}\left(\delta V_{j}\right) \mathrm{d} s \\
= & 0
\end{aligned}
$$

where $\nu_{i}$ are the components of outer unit normal of the deformed boundary surface. We can obtain from eqn (13) the basic governing equations at bifurcation; that is

$$
\delta \dot{T}_{i j, i}=0 \quad \text { in } \quad v
$$

and

$$
\delta \dot{T}_{i j} \nu_{i}=0 \quad \text { on } \quad s_{f}
$$

with the constraint on the remaining part $s_{\nu}$ of the boundary surface known as

$$
\delta V_{i}=0 \quad \text { on } \quad s_{v}
$$


The physical ground of the previous statement on bifurcation is based on the nature of potential energy implicated in the functional II (see HILL [1959]). It provides a sufficient criterion for stability of a system under dead loading. This criterion claims that any geometrically possible disturbance then causes a positive increase of II; that is

$$
\Delta \mathrm{II}>0 .
$$

The statement of eqn (15a) implies that the internal energy stored or dissipated during the disturbance should exceed the work done by the external (dead) load. Expanding $\Delta \mathrm{II}$ into series, to second order, we have

$$
\Delta \mathrm{II}=\delta \mathrm{II}+\frac{1}{2 !} \delta^{2} \mathrm{II} .
$$

Since $\delta \mathrm{II}=0$ at rate equilibrium, the sufficient condition given by eqn (15a) for stability then reduces to ensuring

$$
\delta^{2} \mathrm{II}>0
$$

In this case, the functional II in eqn (1) is not only a stationary point but also a minimum.

$$
\delta^{2} \mathrm{II}=0
$$

then becomes a stability limit or critical condition, which must be associated with the stationary condition given by eqn (11c).

The significance of eqn (16) is also confirmed by its similarity in form and equality in sense with the sufficient condition for uniqueness under the requisite condition of using a linear comparison model in the constitutive description (see HILL [1959] and BURKE \& NIX [1979]). The use of a linear comparison model has the two-fold benefit of both searching for the lowest critical value and guiding the strain path to be single-valued in integration. This "linearizing" device has been extended by LI [1991] to cover the conditions of having plastic dilatancy or strain-softening in materials.

\section{II.2. Dilatational plastic constitutive equations}

In view of the plastic dilatancy caused by voids in ductile materials, a constitutive model was lately developed and justified (LI [1991]; Li et al. [1992]; Liv \& Li [1992]).

According to this theory, the total deformation rate is composed of an elastic part, following Hooke's law, and of a plastic component, including plastic dilatation. Eventually, we have

$$
\begin{aligned}
D_{i j} & =D_{i j}^{(\mathrm{e})}+D_{i j}^{(\mathrm{p})} \\
& =\frac{1}{E}\left[(1+\nu) \frac{\mathcal{D} \tau_{i j}}{\mathcal{D} t}-\nu \delta_{i j} \frac{\mathcal{D} \tau_{k k}}{\mathcal{D} t}\right]+\frac{9}{4 E_{\mathrm{te}}^{(\mathrm{p})}} \frac{S_{i j} S_{k l}}{\sigma_{\mathrm{e}}^{2}} \frac{\mathcal{D} \tau_{k l}}{\mathcal{D} t}+\delta_{i j} \frac{1}{3 E_{\mathrm{tm}}^{(\mathrm{p})}} \frac{\mathcal{D} \tau_{k k}}{\mathcal{D} t},
\end{aligned}
$$

where $E$ and $\nu$ are, respectively, Young's modulus and Poisson's ratio; $\sigma_{\mathrm{e}}=\left(\frac{3}{2} S_{i j} S_{j i}\right)^{1 / 2}$ is equivalent stress with $S_{i j}$ being the deviatoric stress; $\delta_{i j}$ represents Kronecker delta; and $\boldsymbol{E}_{\mathrm{te}}^{(\mathbf{p})}\left(\doteq \mathrm{d} \sigma_{\mathrm{e}} / \mathrm{d} \varepsilon_{\mathrm{e}}\right)$ and $\boldsymbol{E}_{\mathrm{tm}}^{(\mathbf{p})}\left(\dot{=} \mathrm{d} \sigma_{\mathrm{m}} / \mathrm{d} \varepsilon_{\mathrm{m}}\right)$ are the plastic tangent moduli along the equivalent stress-strain curve $\left(\sigma_{\mathrm{e}}-\varepsilon_{\mathrm{e}}\right)$ and the mean stress-strain curve $\left(\sigma_{\mathrm{m}}-\varepsilon_{\mathrm{m}}\right)$, respectively, with the mean stress defined as $\sigma_{\mathrm{m}}=\frac{1}{3}\left(\sigma_{k k}\right)$. 
The inverse form of eqn (18b) can be derived as

$$
\frac{\mathcal{D} \tau_{i j}}{\mathcal{D} t}=L_{i j k l} D_{k l}
$$

and the stiffness tensor

$$
\begin{aligned}
L_{i j k l} & =\frac{E}{(1+\nu)}\left[\frac{1}{2}\left(\delta_{i k} \delta_{j l}+\delta_{i l} \delta_{j k}\right)\right. \\
& \left.+\delta_{i j} \delta_{k l} \frac{\nu-E / 3 E_{\mathrm{tm}}^{(\mathrm{p})}}{1-2 \nu+E / E_{\mathrm{tm}}^{(\mathrm{p})}}-\frac{3}{2 \sigma_{\mathrm{e}}^{2}} \frac{E}{E_{\mathrm{te}}^{(\mathrm{p})}} \frac{S_{i j} S_{k l}}{\frac{2}{3}(1+\nu)+E / E_{\mathrm{te}}^{(\mathrm{p})}}\right] .
\end{aligned}
$$

The advantage of this constitutive model is that one can account for the plastic dilatation without enforcing the material to be strain-softened (i.e. the loading surface must shrink with respect to void growth as that proposed by GuRSON [1977]) in a continuum sense. Physically and usually, the continuum sense of strain-hardening can still prevail in the material being voided until the stage of initial fracture. Secondly, the rate of porosity development (i.e. the increase of void volume fraction $f$ with respect to the change of mean stress), characterized by the plastic tangent modulus $E_{\mathrm{tm}}^{(\mathrm{p})}$, is taken as a governing parameter instead of the void volume fraction itself (see Section V). Finally, as shown by LI and HowARD [1983] and LI et al. [1989], the damage to the secondary voids in the matrix material around the primary voids, initial void shapes (spherical, cylindrical, flat, etc.) and void growth history (larger or smaller porosity at initial stage) are of practical importance. In addition, the pattern of void distribution is also known to be significant. To our knowledge, these effects can be best estimated by experimental evaluation. Therefore, a phenomenological approach given by (18b) and (19b) is still preferable.

The discrepancy between the predictions of Gurson's model and experimental observation has been noticed by MEAR [1990] as being partly due to the fact that Gurson's model does not account for irregular void shape or non-uniform void distribution. The assumptions underlying the constitutive characterization based on Gurson's model are also discussed and questioned by BECKER et al. [1988]. So far, we can follow the conclusion stated by Li et al. [1989]: "Theoretical modelling does help us understand the microstructural mechanism, the damage evolution and the characterizing parameters that should be chosen to describe the process of ductile damage. However, for the sake of making a quantitative estimation of its macroscopic microscopic behaviour, the undetermined effects involved in physical material should be completed and taken into account by employing experimental results." The plastic tangent moduli $E_{\mathrm{te}}^{(\mathrm{p})}$ and $E_{\mathrm{tm}}^{(\mathrm{p})}$ in eqn $(18 \mathrm{~b})$ can meet this need. These parameters are phenomenological in form, yet they are able to take into account the effects of microstructural evolution. Their determination has been exemplified by LI et al. [1992] and also will be shown later in this paper.

\section{SOLUTIONS FOR SHEAR-BAND BIFURCATION}

\section{III.1. Localized mode}

In the Cartesian coordinates, the plane-strain components of velocity and their gradients can be expressed, respectively, as (HILL \& HuTCHINSON [1975]) 


$$
\begin{gathered}
\delta V_{i}=V_{i}(n) \quad(i=1,2), \\
\delta V_{i, j}=\frac{\mathrm{d} V_{i}}{\mathrm{~d} n} n_{j}=c_{i} n_{j} \quad(j=1,2) .
\end{gathered}
$$

In eqn (20b), $n_{j}$ are the components of the normal $n$ projecting along the coordinate axes; that is

$$
n=n_{i} x_{i} \quad(i=1,2)
$$

where $n_{1}=\cos \alpha, n_{2}=\sin \alpha$, and $\alpha$ is the angle between the normal $n$ of the band and the axis $x_{1}$.

Under the condition of uniaxial loading by uniform stress $\sigma_{11}=\sigma$, bifurcation equations can be obtained by employing eqn (13) or eqn (14a) and the corresponding eqns (5), (6) and (7). In the end we have

$$
\begin{gathered}
L_{1111}\left(\delta V_{1,1}\right)_{1}+L_{1122}\left(\delta V_{2,2}\right)_{, 1}+L_{1212}\left(\delta V_{1,2}+\delta V_{2,1}\right)_{, 2}-\sigma\left(\delta V_{1,1}\right)_{, 1} \\
-(\sigma / 2)\left(\delta V_{2,1}+\delta V_{1,2}\right)_{, 2}=0 \\
L_{2222}\left(\delta V_{2,2}\right)_{, 2}+L_{1122}\left(\delta V_{1,1}\right)_{, 2}+L_{1212}\left(\delta V_{2,1}+\delta V_{1,2}\right)_{, 1}+(\sigma / 2)\left(\delta V_{2,1}-\delta V_{1,2}\right)_{, 1}=0
\end{gathered}
$$

Making use of eqn (20) and taking

$$
r=c_{1} / c_{2} \quad \text { and } \quad m=n_{2} / n_{1},
$$

we can obtain from eqns (22a) and (22b)

$$
\begin{gathered}
\left(L_{1212}-\frac{\sigma}{2}\right) m^{4}+\frac{1}{L_{2222}}\left[\left(L_{1111}-\sigma\right) L_{2222}+\left(L_{1212}^{2}-\frac{\sigma^{2}}{4}\right)\right. \\
\left.-\left(L_{1212}+L_{1122}-\frac{\sigma}{2}\right)^{2}\right] m^{2}+\frac{L_{1111}-\sigma}{L_{2222}}\left(L_{1212}+\frac{\sigma}{2}\right)=0 \\
r=-\frac{L_{2222} m^{2}+\left(L_{1212}+\frac{\sigma}{2}\right)}{\left(L_{1212}+L_{1122}-\frac{\sigma}{2}\right) m} .
\end{gathered}
$$

The problem now is reduced to searching for the real roots of $m$ in eqn (23a). The evaluation of the components of stiffness tensor included in eqns (23a) and (23b) can be carried out through using eqn (19b), which is dependent on the pre-bifurcation stress states (e.g. plane stress, plane strain). If incompressibility is imposed on the bifurcation mode, then

$$
c_{1} n_{1}+c_{2} n_{2}=0 \quad \text { or } \quad r=-m \text {. }
$$

In this case, eqns (23a) and (23b) can be combined into a single fourth-order algebraic equation, which can yield exactly the result given by HILL and HuTCHINSON [1975] for plane-strain stress state; that is 


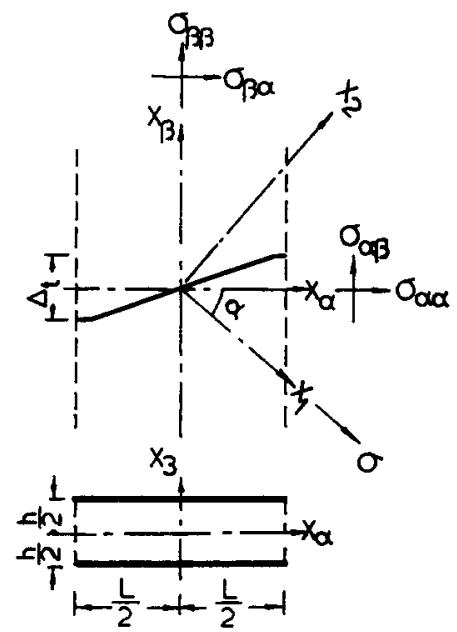

Fig. 1. The $\left(x_{\alpha}, x_{\beta}\right)$ coordinate system.

$$
\left(L_{1212}-\frac{\sigma}{2}\right) m^{4}+\left(L_{1111}+L_{2222}-2 L_{1122}-2 L_{1212}\right) m^{2}+\left(L_{1212}+\frac{\sigma}{2}\right)=0,
$$

where the sum of $\left(L_{1111}+L_{2222}-2 L_{1122}\right)$ and the term $L_{1212}$ can be made, respectively, equivalent to the parameters $4 \mu^{*}$ and $\mu$ of HiLL and HutCHINSON [1975].

\section{III.2. Diffuse mode}

Neither the width of band nor the actual distribution of velocity variation appear in the localized mode stated previously. Therefore, we need a diffuse mode of analysis to introduce these factors into the solution.

Figure 1 demonstrates a band having a width $L$ and lying in a sheet of thickness $h$. The band is parallel to the axis called $x_{\beta}$ and has a normal in the direction of the axis $x_{\alpha}$. The angle between $x_{\alpha}$ and the uniaxial loading direction $x_{1}$ will be determined as $\alpha$. Therefore, the pre-bifurcation stress state associated with the $\left(x_{\alpha}, x_{\beta}\right)$ coordinates can be correlated to the uniaxial stress $\sigma$ as

$$
\begin{aligned}
& \sigma_{\alpha \alpha}=\sigma \cos ^{2} \alpha, \\
& \sigma_{\beta \beta}=\sigma \sin ^{2} \alpha \\
& \sigma_{\alpha \beta}=\sigma_{\beta \alpha}=\sigma \sin \alpha \cos \alpha, \\
& \sigma_{3 \alpha}=\sigma_{\beta 3}=\sigma_{33}=0 .
\end{aligned}
$$

The bifurcation mode, in terms of the variation of velocity, is prescribed such that

$$
\delta V_{\alpha, \beta}=\delta V_{\beta, \beta}=\delta V_{3, \alpha}=\delta V_{3, \beta}=\delta V_{3,3}=0
$$

Besides, the mode should satisfy the following boundary conditions: 


$$
\begin{aligned}
& \delta V_{\alpha}=0 \quad \text { and } \delta D_{3 \alpha}=0 \quad \text { at } x_{\alpha}=0 \text {, } \\
& \delta V_{3}=0 \quad \text { and } \delta D_{3 \alpha}=0 \quad \text { at } x_{3}=0 \text {, } \\
& \int_{-h / 2}^{h / 2} \delta \dot{T}_{\alpha 3} \mathrm{~d} x_{3}=0 \text { and } \int_{-h / 2}^{h / 2} \delta \dot{T}_{\alpha \alpha} \mathrm{d} x_{3}=0 \text { at } x_{\alpha}=L / 2 \text {, } \\
& \delta \dot{T}_{3 \alpha}=0 \quad \text { and } \delta \dot{T}_{33}=0 \quad \text { at } x_{3}=h / 2 \text {. }
\end{aligned}
$$

In eqns (26b), $\delta D_{3 \alpha}=\frac{1}{2}\left(\delta V_{3, \alpha}+\delta V_{\alpha, 3}\right)$.

The variation of nominal stress rate can be expressed as

$$
\begin{aligned}
\delta \dot{T}_{\alpha \alpha} & =\left(L_{\alpha \alpha \alpha \alpha}-\sigma_{\alpha \alpha}\right) \delta V_{\alpha, \alpha}+\left(L_{\alpha \alpha \alpha \beta}-\sigma_{\alpha \beta}\right) \delta V_{\beta, \alpha} \\
\delta \dot{T}_{\beta \beta} & =L_{\alpha \alpha \beta \beta} \delta V_{\alpha, \alpha}+L_{\beta \beta \alpha \beta} \delta V_{\beta, \alpha} \\
\delta \dot{T}_{33} & =L_{\alpha \alpha 33} \delta V_{\alpha, \alpha}+L_{33 \alpha \beta} \delta V_{\beta, \alpha} \\
\delta \dot{T}_{\alpha 3} & =\left(L_{\alpha 3 \alpha 3}-\frac{\sigma_{\alpha \alpha}}{2}\right) \delta V_{\alpha, 3}-\frac{\sigma_{\alpha \beta}}{2} \delta V_{\beta, 3}
\end{aligned}
$$

and $\delta \dot{T}_{3 \alpha}=\delta \dot{T}_{\alpha 3}$ since $\delta V_{3, \alpha}=0$.

Let the variation of velocity take the form of

$$
\begin{aligned}
\delta V_{\alpha} & =\sin \frac{\pi x_{\alpha}}{L} \phi \Delta_{2}, \\
\delta V_{\beta} & =\sin \frac{\pi x_{\alpha}}{L} \phi \Delta_{4} \\
\delta V_{3} & =0 \\
\phi & =1+(1-\omega) \cos \frac{2 \pi x_{3}}{h}-\omega \cos \frac{4 \pi x_{3}}{h} .
\end{aligned}
$$

Here, $\Delta_{2}$ and $\Delta_{4}$ are two generalized velocity components.

The shape function across the thickness is formulated as $\phi$, in which the factor $\omega$ can be functioned as an adjustable parameter in order to yield the lowest critical stress. It is not difficult to verify that all the prerequisite conditions listed in eqns (26a) and (26b) can be exactly satisfied by the bifurcation pattern formulated in eqn (28).

Substituting (28) into (11a) and making some mathematical manipulations, gives the second variational function $Q$, which is

$$
\begin{aligned}
Q & =\left(L_{\alpha \alpha \alpha \alpha}-\sigma_{\alpha \alpha}\right) \frac{\pi^{2}}{16} \frac{h}{L}\left(\frac{3}{2}-\omega+\omega^{2}\right) \Delta_{2}^{2} \\
& +\left(L_{\alpha \alpha \alpha \beta}-\sigma_{\alpha \beta}\right) \frac{\pi^{2}}{8} \frac{h}{L}\left(\frac{3}{2}-\omega+\omega^{2}\right) \Delta_{2} \Delta_{4} \\
& +\left(L_{\alpha \beta \alpha \beta}+\frac{\sigma_{\alpha \alpha}}{2}-\frac{\sigma_{\beta \beta}}{2}\right) \frac{\pi^{2}}{16} \frac{h}{L}\left(\frac{3}{2}-\omega+\omega^{2}\right) \Delta_{4}^{2} \\
& +\left(L_{\alpha 3 \alpha 3}-\frac{\sigma_{\alpha \alpha}}{2}\right) \frac{\pi^{2}}{8} \frac{L}{h}\left(1-2 \omega+5 \omega^{2}\right) \Delta_{2}^{2} \\
& +\left(L_{\beta 3 \beta 3}-\frac{\sigma_{\beta \beta}}{2}\right) \frac{\pi^{2}}{8} \frac{L}{h}\left(1-2 \omega+5 \omega^{2}\right) \Delta_{4}^{2} \\
& -\sigma_{\alpha \beta} \frac{\pi^{2}}{8} \frac{L}{h}\left(1-2 \omega+5 \omega^{2}\right) \Delta_{2} \Delta_{4}
\end{aligned}
$$

where $h / L$ (or its inverse $L / h$ ) is a geometrical aspect parameter of the band.

Owing to the stationary condition of eqn (11c), that should be satisfied at bifurcation, 


$$
\frac{\partial Q}{\partial \Delta_{2}}=0, \quad \frac{\partial Q}{\partial \Delta_{4}}=0 .
$$

A set of homogeneous and linear equations can be obtained from eqns $(30 \mathrm{a}, \mathrm{b})$ for solving the problem and takes the form:

$$
\begin{aligned}
& C_{1} \Delta_{2}+C_{2} \Delta_{4}=0, \\
& C_{2} \Delta_{2}+C_{3} \Delta_{4}=0,
\end{aligned}
$$

where

$$
\begin{aligned}
C_{1} & =\left(L_{\alpha \alpha \alpha \alpha}-\sigma_{\alpha \alpha}\right) \frac{\pi^{2}}{8} \frac{h}{L}\left(\frac{3}{2}-\omega+\omega^{2}\right) \\
& +\left(L_{\alpha 3 \alpha 3}-\frac{\sigma_{\alpha \alpha}}{2}\right) \frac{\pi^{2}}{4} \frac{L}{h}\left(1-2 \omega+5 \omega^{2}\right), \\
C_{2} & =\left(L_{\alpha \alpha \alpha \beta}-\sigma_{\alpha \beta}\right) \frac{\pi^{2}}{8} \frac{h}{L}\left(\frac{3}{2}-\omega+\omega^{2}\right)-\sigma_{\alpha \beta} \frac{\pi^{2}}{8} \frac{L}{h}\left(1-2 \omega+5 \omega^{2}\right) \\
C_{3} & =\left(L_{\alpha \beta \alpha \beta}+\frac{\sigma_{\alpha \alpha}}{2}-\frac{\sigma_{\beta \beta}}{2}\right) \frac{\pi^{2}}{8} \frac{h}{L}\left(\frac{3}{2}-\omega+\omega^{2}\right) \\
& +\left(L_{\beta 3 \beta 3}-\frac{\sigma_{\beta \beta}}{2}\right) \frac{\pi^{2}}{4} \frac{L}{h}\left(1-2 \omega+5 \omega^{2}\right) .
\end{aligned}
$$

Shear-band bifurcation occurs at the point where the determinant

$$
D=C_{1} C_{3}-C_{2}^{2}=0 .
$$

Hereafter, once the material parameters concerned are determined, then it is only a matter of using a computer to seek for appropriate values of the angle $\alpha$ and of the adjustable parameter $\omega$ so as to yield the lowest critical stress that corresponds to each assigned value for the geometrical aspect parameter $h / L$ of the band.

\section{CALCULATiOn OF RESUlts}

For pre-bifurcation stress state, we choose

(a) plane-stress condition: $\sigma_{33}=\mathbf{0}$;

(b) plane-strain condition: $\sigma_{33}=\zeta \sigma$;

and according to the deformation theory of plasticity, it is not difficult to determine that

$$
\zeta=(\nu+\psi / 3) /(1+2 \psi / 3), \quad \psi=\frac{3}{2} \frac{E}{E_{\mathrm{se}}}-(1+\nu)
$$

where $E_{\mathrm{se}}$ is the secant modulus taken from the equivalent stress-strain curve of the material used. Throughout the following calculations, we define a yield strain parameter as

$$
\varepsilon_{\mathrm{y}}=\sigma_{\mathrm{y}} / E \quad(=0.002 \text { for steel }),
$$

where $\sigma_{\mathrm{y}}$ is the yield stress. 


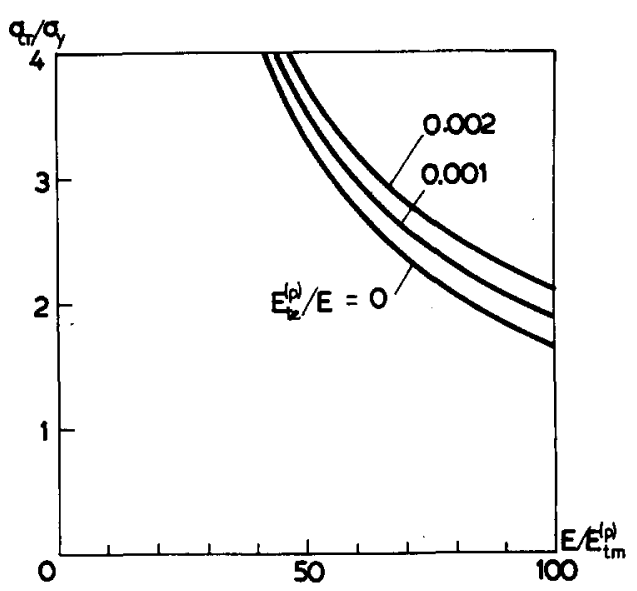

Fig. 2. The critical stress $\sigma_{\mathrm{cr}} / \sigma_{\mathrm{y}}$ varying with respect to plastic dilatancy $E / E_{\mathrm{tm}}^{(\mathrm{p})}$ in the localized-mode solution with the plane-stress condition for the pre-bifurcation stress state $\left(\nu=0.3, \varepsilon_{\mathrm{y}}=0.002\right)$.

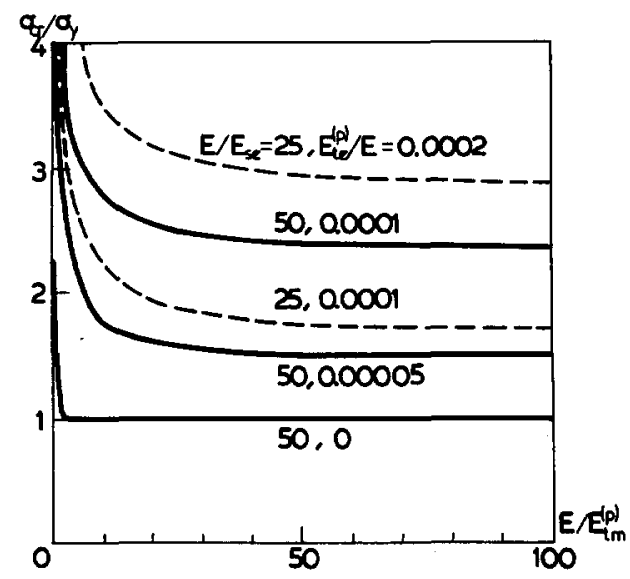

Fig. 3. The critical stress $\sigma_{\mathrm{cr}} / \sigma_{\mathrm{y}}$ varying with respect to plastic dilatancy $E / E_{\mathrm{tm}}^{(\mathrm{p})}$ in the localized-mode solution with the plane-strain condition for the pre-bifurcation stress state $\left(\nu=0.3, \varepsilon_{\mathrm{y}}=0.002\right)$.

\section{IV.1. Solution of localized-mode bifurcation}

Figure 2 shows the distribution of critical stress $\sigma_{\mathrm{cr}}$ (normalized by the yield stress $\sigma_{\mathrm{y}}$ ) with respect to a plastic dilatancy parameter $E / E_{\mathrm{tm}}^{(\mathrm{p})}$, under the uniaxial tensile loading of a plane-stress condition with various strain-hardening behavior characterized by the normalized tangent modulus $E_{\mathrm{te}}^{(\mathrm{p})} / E=0,0.001$ and 0.002 . Here, any pair of values assigned for $E_{\mathrm{te}}^{(\mathrm{p})} / E$ and $E / E_{\mathrm{tm}}^{(\mathrm{p})}$ are taken as associated with the loading stress $\sigma$ attained at that critical moment. It is clearly seen from the demonstration of this figure that the critical stress is very sensitive to the plastic dilatancy; no reasonable value for critical stress can be obtained without taking into account this effect. The bifurcation angle $\alpha$ varies between $2.5^{\circ}$ and $5.9^{\circ}$ within the scope of calculations. This implies that the very initial shear band could be lying nearly perpendicular to the uniaxial loading direction.

The critical stress would be much more sensitive to plastic dilatancy, if the prebifurcation stress is in a plane-strain condition. As shown in Fig. 3, a slight increase of 
plastic dilatancy, say if $E / E_{\mathrm{tm}}^{(\mathrm{p})}$ changes from 0 to 10 , may decrease enormously the loading level at bifurcation. The results calculated and displayed in this figure cover a scope of parameters as $E / E_{\mathrm{se}}=25,50$ and $E_{\mathrm{te}}^{(\mathrm{p})} / E=0,5 \times 10^{-5}, 1 \times 10^{-4}$ and $2 \times 10^{-4}$. They also indicate that a soft material response is needed for the plane-strain condition to trigger bifurcation; here, the normalized tangent modulus $E_{\mathrm{te}}^{(\mathrm{p})} / E$ is one order softer than those of the plane-stress case. Another striking feature is that the bifurcation angle $\alpha$ varies between $44.7^{\circ}$ and $45.1^{\circ}$.

\section{IV.2. Solution of diffuse-mode bifurcation}

Figure 4 (a) shows the distribution of critical stress $\alpha_{\mathrm{cr}}$ with respect to the geometrical aspect parameter $h / L$ under the plane-stress loading condition. The material parameters are chosen as $\nu=0.3, \varepsilon_{\mathrm{y}}=0.002, E_{\mathrm{te}}^{(\mathrm{p})} / E=0$ or 0.001 and $E / E_{\mathrm{tm}}^{(\mathrm{p})}=70$ or 100 . Each curve tends to its own corresponding result of the localized-mode solution (abbreviated to ls). The bifurcation angle $\alpha$ varies between $0^{\circ}$ and $4.8^{\circ}$, and follows a similar trend to the localized-mode solution.

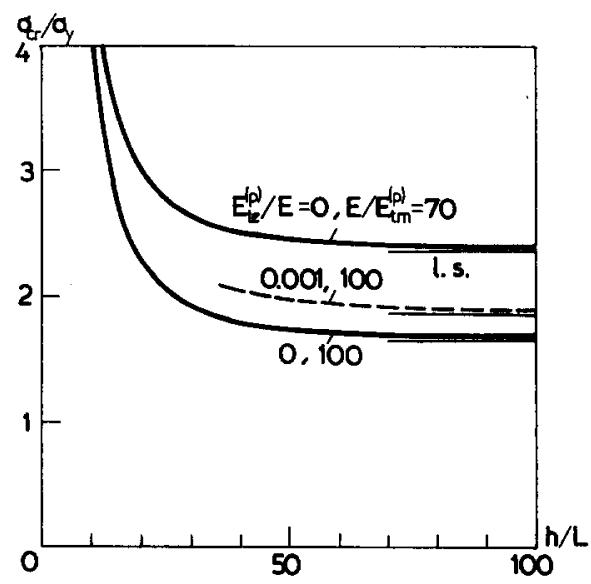

(a)

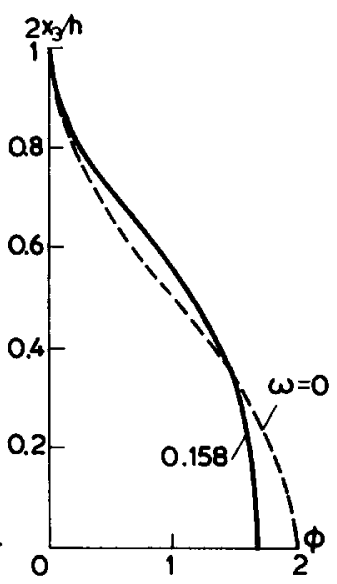

(b)

Fig. 4. (a) The critical stress $\sigma_{\mathrm{cr}} / \sigma_{\mathrm{y}}$ varying with respect to the geometrical aspect parameter $h / L$ in the diffuse-mode solution $\left(E_{\mathrm{te}}^{(\mathrm{p})} / E=0, \nu=0.3, \varepsilon_{\mathrm{y}}=0.002\right)$ with the plane-stress condition for the prebifurcation stress state. (b) The distribution of the shape function $\phi$.

Table 1. The critical stress $\sigma_{\mathrm{cr}} / \sigma_{\mathrm{y}}$ varying with respect to the geometrical aspect parameter $h / \mathrm{L}$ and plastic dilatancy $E / E_{\mathrm{tm}}^{(\mathrm{p})}\left(E_{\mathrm{te}}^{(\mathrm{p})} / E=0, \nu=0.3, \varepsilon_{\mathrm{y}}=0.002\right)$

\begin{tabular}{|c|c|c|c|c|c|c|c|}
\hline \multirow{3}{*}{$\frac{E / E_{\mathrm{tm}}^{(\mathrm{p})}}{60}$} & \multicolumn{6}{|c|}{$\sigma_{\mathrm{cr}} / \sigma_{\mathrm{y}}$} & \multirow{3}{*}{$\begin{array}{c}\text { 1s } \\
2.7495\end{array}$} \\
\hline & \multicolumn{2}{|c|}{$h / L=10^{2}$} & \multicolumn{2}{|c|}{$h / L=10^{3}$} & \multicolumn{2}{|c|}{$h / L=10^{4}$} & \\
\hline & $\begin{array}{c}2.7755 \\
(\omega=0)\end{array}$ & $\begin{array}{r}2.7726 \\
(0.159)\end{array}$ & $\begin{array}{c}2.7498 \\
(0)\end{array}$ & $\begin{array}{c}2.7498 \\
(0)\end{array}$ & $\begin{array}{l}2.7495 \\
(0)\end{array}$ & $\begin{array}{c}2.7495 \\
(0)\end{array}$ & \\
\hline 80 & $\begin{array}{c}2.0933 \\
(\omega=0)\end{array}$ & $\begin{array}{l}2.0904 \\
(0.158)\end{array}$ & $\begin{array}{c}2.0677 \\
(0)\end{array}$ & $\begin{array}{c}2.0677 \\
(0)\end{array}$ & $\begin{array}{c}2.0674 \\
(0)\end{array}$ & $\begin{array}{c}2.0674 \\
(0)\end{array}$ & 2.0674 \\
\hline 100 & $\begin{array}{c}1.6823 \\
(\omega=0)\end{array}$ & $\begin{array}{r}1.6794 \\
(0.158)\end{array}$ & $\begin{array}{c}1.6568 \\
(0)\end{array}$ & $\begin{array}{r}1.6567 \\
(0.121)\end{array}$ & $\begin{array}{c}1.6565 \\
(0)\end{array}$ & $\begin{array}{c}1.6565 \\
(0)\end{array}$ & 1.6565 \\
\hline
\end{tabular}


Table 1 lists the calculated data for much larger values of $h / L$ to show the asymptotic trend (with $\nu=0.3, \varepsilon_{\mathrm{y}}=0.002, E_{\mathrm{te}}^{(\mathrm{p})} / E=0$ and $E / E_{\mathrm{tm}}^{(\mathrm{p})}=60,80$ and 100). The results clearly indicate that once the geometrical aspect parameter $h / L$ approaches $10^{4}$, the critical stresses are the same to four decimal points as those of the localized-mode solution.

The function of selecting an adjustable parameter is noticeable for $h / L \leq 10^{2}$ but becomes immaterial when $h / L$ approaches $10^{3}$. The shape function $\phi$ is delineated in Fig. 4(b) for $\omega=0$ and 0.158 , spreading over the half thickness of the sheet.

Substituting (28) into (27), eventually, we obtain the variations of the nominal stress rates (normalized by yield stress $\sigma_{\mathrm{y}}$ ) at the moment of bifurcation in the form of

$$
\begin{aligned}
\frac{\delta \dot{T}_{\alpha \alpha}}{\sigma_{\mathrm{y}}} & =T_{\alpha}\left(\cos \frac{\pi x_{\alpha}}{L} \phi\right) \gamma \\
\frac{\delta \dot{T}_{\beta \beta}}{\sigma_{\mathrm{y}}} & =T_{\beta}\left(\cos \frac{\pi x_{\alpha}}{L} \phi\right) \gamma \\
\frac{\delta \dot{T}_{33}}{\sigma_{\mathrm{y}}} & =T_{3}\left(\cos \frac{\pi x_{\alpha}}{L} \phi\right) \gamma
\end{aligned}
$$

Here

$$
\begin{gathered}
T_{\alpha}=\frac{\xi}{\sigma_{\mathrm{y}}}\left[L_{\alpha \alpha \alpha \alpha}-\sigma_{\alpha \alpha}+\rho\left(L_{\alpha \alpha \alpha \beta}-\sigma_{\alpha \beta}\right)\right] \\
T_{\beta}=\frac{\xi}{\sigma_{\mathrm{y}}}\left(L_{\alpha \alpha \beta \beta}+\rho L_{\beta \beta \alpha \beta}\right) \\
T_{3}=\frac{\xi}{\sigma_{\mathrm{y}}}\left(L_{\alpha \alpha 33}+\rho L_{33 \alpha \beta}\right) \\
\xi=\frac{1}{2 \rho(1-\omega)} \\
\rho=\Delta_{4} / \Delta_{2} .
\end{gathered}
$$

In eqn (36), $\gamma$ refers to the shear rate that occurs in the band at the middle plane $\left(x_{3}=0\right)$ of the plane sheet, since

$$
\gamma=\Delta_{t} / L
$$

As specified by Fig. 1,

$$
\Delta_{t}=\left.2 \delta V_{\beta}\right|_{\left(x_{\alpha}=L / 2, x_{3}=0\right)}
$$

where $\Delta_{t}$ denotes the rate of mutual displacement between the two border lines of the band lying along the $x_{\beta}$ direction and spanning a width of $L$ in the plane at $x_{3}=0$. In order to understand the triaxiality state at bifurcation, we can calculate that

$$
\begin{aligned}
\frac{\delta \dot{T}}{\sigma_{\mathrm{y}}} & =\frac{1}{3 \sigma_{\mathrm{y}}}\left(\delta \dot{T}_{\alpha \alpha}+\delta \dot{T}_{\beta \beta}+\delta \dot{T}_{33}\right)=T\left(\cos \frac{\pi x_{\alpha}}{L} \phi\right) \gamma \\
T & =\frac{1}{3}\left(T_{\alpha}+T_{\beta}+T_{3}\right) .
\end{aligned}
$$


Table 2. The stress rates $T_{3}$ and $T$ varying with respect to the geometrical aspect parameter $h / L$ and plastic dilatancy $E / E_{\mathrm{tm}}^{(\mathrm{p})}\left(E_{\mathrm{te}}^{(\mathrm{p})} / E=0, \nu=0.3, \varepsilon_{\mathrm{y}}=0.002\right)$

\begin{tabular}{rccccccc}
\hline & \multicolumn{3}{c}{$T_{3}$} & & \multicolumn{3}{c}{$T$} \\
\cline { 2 - 3 } \cline { 6 - 8 }$E / E_{\mathrm{tm}}^{(\mathrm{p})}$ & $h / L=10^{2}$ & $h / L=10^{3}$ & $h / L=10^{4}$ & & $h / L=10^{2}$ & $h / L=10^{3}$ & $h / L=10^{4}$ \\
\hline 60 & 23.130 & 19.388 & 19.239 & & 10.538 & 8.818 & 8.631 \\
80 & 20.068 & 16.911 & 16.738 & & 9.158 & 7.772 & 7.560 \\
100 & 17.949 & 17.159 & 15.199 & & 8.170 & 7.835 & 7.026 \\
\hline
\end{tabular}

Table 2 presents the variation of $T_{3}$ and $T$ with various plastic dilatancy conditions of $E / E_{\mathrm{tm}}^{(\mathrm{p})}$ and geometrical aspect parameters $h / L$. The calculations, for these nominal stress rates triggered at bifurcation, predict a large normal stress rate $\delta \dot{T}_{33}$ at the middle plane $\left(\dot{x}_{3}=0\right)$ of the sheet, although the pre-bifurcation stress state is of the planestress condition. The high degree of tensile triaxiality quantified by the positive value of $T$ furnishes the mechanical basis for incurring extra void nucleation/growth within the band region, and also, the shape function $\phi$ indicates that the triaxiality state is most serious at the middle plane $\left(x_{3}=0\right)$ of sheet and can be much lessened by reaching to the surface plane. This prediction explains the experimental phenomenon shown later in Fig. 6.

\section{COMPARISON WITH EXPERIMENTS AND DISCUSSIONS}

The material used for this purpose is a dual-phase steel, whose chemical composition $(\mathrm{wt} \%)$ is $\mathrm{C}-0.08, \mathrm{Mn}-1.50, \mathrm{Si}-0.30, \mathrm{P}-0.008, \mathrm{~S}-0.010$; the remainder is Fe. The volume fraction of martensite is $18 \%$. The yield stress $\sigma_{\mathrm{y}}$ and tensile strength $\sigma_{\mathrm{b}}$ of this steel are $370 \mathrm{MPa}$, and $650 \mathrm{MPa}$, respectively.

An in situ uniaxial tensile test for observation was carried out by using a Hitachi S-570 scanning electron microscope (SEM). The shape and size of the specimens tested are shown in Fig. 5. A gauge length of $L_{0}=6.5 \mathrm{~mm}$ was set in the middle part of each specimen, by lightly pre-marking two straight lines on the specimen surface in the direction perpendicular to the tensile axis, in order to measure the relative displacement $\Delta L$ and the corresponding nominal strain $\Delta L / L_{0}$.

The loading procedure was controlled manually and at a slow rate. As for each test, loading was interrupted and then kept constant at several intervals to record the development of deformations, by measuring the current gauge length $L_{\mathrm{c}}$, width $w$ and thickness $h$ of the specimen being tested. After shear banding, each specimen could only be pulled to a certain extent of $\Delta L / L_{0}\left(=L_{\mathrm{c}} / L_{\mathrm{o}}-1\right)$ and then completely unloaded to free all the loading stress, so as to measure carefully the geometry and

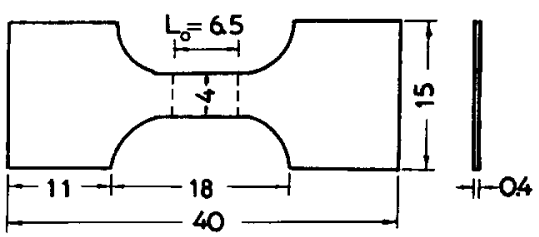

Fig. 5. The geometrical size and shape of the specimen. 
internal porosity of the material. All the parameters measured are associated with only that particular stage of elongation characterized by the nominal strain $\Delta L / L_{0}$. Therefore, besides the specimens being tested for determination of the initiation of the shear band at $\Delta L / L_{0}=0.105$, which is the point of having the maximum loading stress, five more specimens were used to provide the experimental information needed for different elongations (at $\Delta L / L_{0}=0.127,0.159,0.195,0.243$ and 0.296 ) within the whole stage of shear-band localization until the final rupture.

The average true strains along the width and the thickness directions of each specimen are, respectively, denoted as $\varepsilon_{2}$ and $\varepsilon_{3}$. They are evaluated by

$$
\begin{aligned}
& \varepsilon_{2}=\ln \left(w / w_{0}\right), \\
& \varepsilon_{3}=\ln \left(h / h_{0}\right),
\end{aligned}
$$

where $w_{0}$ and $h_{0}$ are the initial width and thickness, respectively. Once shear band occurs, the current width $w$ and current thickness $h$ are measured at the location of band localization.

Void volume fraction $f$ was measured manually and checked by using quantitative image analysis. The initial porosity caused by material imperfection has been discarded from the evaluation; therefore, the results are referred to as the pure growth of porosity with regard to the elongation of material. To perform the measurement, tiny pieces of material were taken from the shear-band zone, either from the surface or the interior of the specimen. In the latter case one-third thickness of the small test piece was ground off from the surface to reveal the internal status of porosity. The determination of taking one-third thickness off was based on both a theoretical consideration (the absolute value associated with the site at one-third off must be near to that at one-half off from the surface, according to the distribution of the shape function $\phi$ in eqn (28)) and for the sake of the practical handling involved in test piece preparation. Since stress unloading occurs in the part of the material away from the shear band, there is no need to be concerned about the evolution of damage in that region. The void volume fraction $f$ measured with respect to increasing nominal strain $\Delta L / L_{0}$ is demonstrated in Fig. 6. There is a sharp turn at the occurrence of the shear band around $\Delta L / L_{0}=0.10$. The interior porosity is much larger than that on the surface.

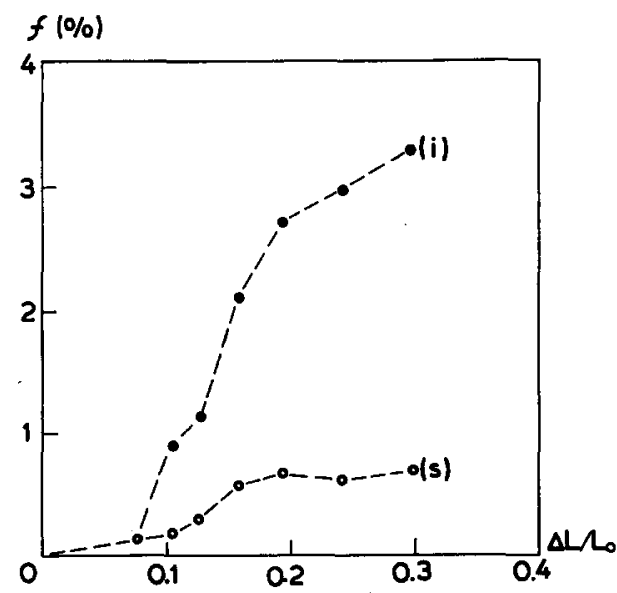

Fig. 6. The void volume fraction $f$ in the shear band vs the nominal strain $\Delta L / L_{0}$. 


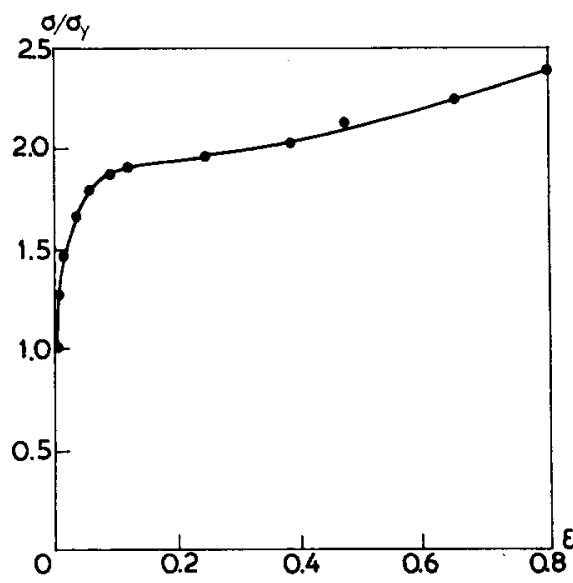

(a)

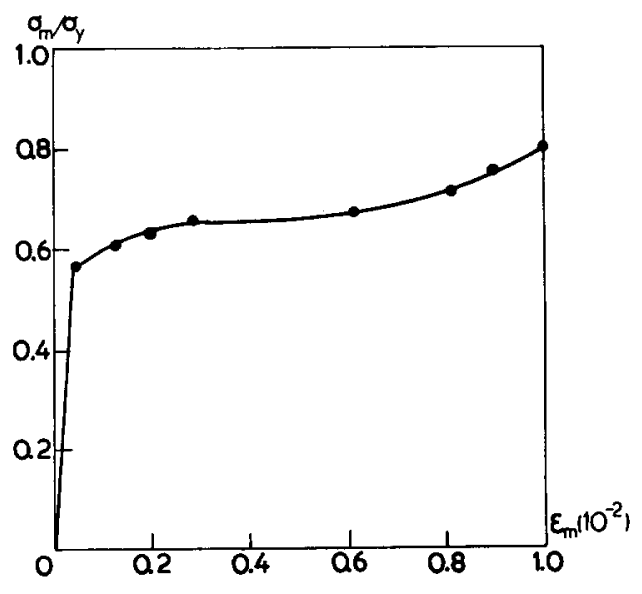

(b)

Fig. 7. The stress-strain curves of the dual-phase steel.

In order to determine the axial true strain $\varepsilon_{1}$ averaged across the width $w$, we make use of the formula

$$
\varepsilon_{1}=f_{\mathrm{a}}-\left(\varepsilon_{2}+\varepsilon_{3}\right),
$$

where $f_{\mathrm{a}}$ should be the average void volume fraction existing in the shear-band zone. We also define that the mean plastic strain caused by voiding is

$$
\varepsilon_{\mathrm{m}}=\left(\varepsilon_{1}+\varepsilon_{2}+\varepsilon_{3}\right) / 3=f_{\mathrm{a}} / 3 .
$$

The rate of porosity development can then be characterized by the increase of mean strain $\varepsilon_{\mathrm{m}}$ with respect to the change of mean stress $\sigma_{\mathrm{m}}$.

Significant necking is not seen until $\Delta L / L_{0} \doteq 0.10$. It practically coincides with the initial appearance of the shear band on the surface and the point of the acceleration of voiding shown in Fig. 6. Eventually, we take the experimental value of critical nominal strain $\left(\Delta L / L_{0}\right)_{\mathrm{cr}}=0.105$, based on an average of six pieces of tests. After some manipulations, we further obtain the curve of uniaxial stress $\sigma_{1}$ (normalized by $\sigma_{\mathrm{y}}$ ) vs uniaxial strain $\varepsilon_{1}$ (or simply $\sigma-\varepsilon$ and in this case it can be used as the equivalent stress-strain curve $\left.\sigma_{\mathrm{e}}-\varepsilon_{\mathrm{e}}\right)$ and of the mean stress $\sigma_{\mathrm{m}}\left(=\sigma / 3\right.$, also normalized by $\left.\sigma_{y}\right)$ vs mean strain $\varepsilon_{\mathrm{m}}$ (i.e. $\sigma_{\mathrm{m}}-\varepsilon_{\mathrm{m}}$ ). All are in the sense of being the true quantity, since they are based on the current dimensions, and are delineated in Figs 7(a) and (b), where the solid circles denote either the point of load interruption (without unloading) before necking or the point of complete unloading after necking. The last five data, after necking, in the Figs 7(a) and (b) can only be obtained by using five separate specimens made of the same material. Following these curves, we can determine the tangent moduli $E_{\mathrm{te}}^{(\mathrm{p})}$ and $E_{\mathrm{tm}}^{(\mathrm{p})}$ in eqn (19) and also the pre-bifurcation stress state needed in bifurcation analysis.

Table 3 presents the critical values calculated for the dual-phase steel sheet tested. Calculations are issued by the localized-mode analysis, since it can be taken as the asymptotic result of the diffuse-mode analysis stated previously. The results of $\sigma_{\mathrm{cr}}, \varepsilon_{\mathrm{cr}}$ and $\left(\Delta L / L_{0}\right)_{\mathrm{cr}}$ are the critical values of $\sigma, \varepsilon$ and $\Delta L / L_{0}$, respectively. Both plane- 
Table 3. The critical values calculated for a dual-phase steel sheet

\begin{tabular}{lccccccc}
\hline $\begin{array}{l}\text { Pre-bifurcation } \\
\text { stress state }\end{array}$ & $E_{\mathrm{te}}^{(\mathrm{p})} / E$ & $E / E_{\mathrm{tm}}^{(\mathrm{p})}$ & $\sigma_{\mathrm{cr}} / \sigma_{\mathrm{y}}$ & $\varepsilon_{\mathrm{cr}}$ & $\left(\Delta L / L_{0}\right)_{\mathrm{cr}}$ & $m$ & $\alpha^{(0)}$ \\
\hline Plane-strain & 0.0001 & 30 & 1.89 & 0.12 & 0.108 & 0.982 & 44 \\
Plane-stress & 0.0008 & 93 & 1.97 & 1.25 & 0.130 & 0.061 & 4 \\
\hline
\end{tabular}

stress and plane-strain stress states are taken into account as two typical conditions of pre-bifurcation. The actual stress state across the thickness of the specimen must be between these two limiting cases. Therefore we can take the critical values 0.108 and 0.130 given in Table 3 for the nominal strains at bifurcation as their bound values. The real prediction should be somewhere between these values and is quite close to the experimental data giving $\left(\Delta L / L_{0}\right)_{\mathrm{cr}}=0.105$. The difference between the critical stresses predicted and tested is small.

So far, it is difficult to justify the theoretical prediction for the bifurcation angle $\alpha$. One of the reasons is that the velocity in the band has its strongest variation (as shown by the diffuse-mode analysis) at the middle plane of the sheet and is not able to be observed initially. Secondly, once seen on the surface, it is shaped like a local bright spot without obvious inclination. Afterwards, cross lines as bands spread out from this spot with their normals lying between $25-30^{\circ}$ with respect to the loading axis. Should these be bands at bifurcation or rather the phenomenon of post-bifurcation? This is still an open question.

Our experiments indicated that only $1.5 \%$ of porosity existed in the material at bifurcation. This fact proves that the dilatational plastic model in eqns (19a) and (19b) provides a good possibility for predicting shear-band bifurcation at a low degree of strain-hardening characterized by $E_{\mathrm{te}}^{(\mathrm{p})} / E$ and small amounts of porosity.

\section{CONCLUSIONS}

The main findings of our research can be summarized as follows:

1. Practically speaking, shear-band bifurcation occurs at the maximum point of uniaxial loading in our experiments. The formation of shear bands takes a process to develop from the interior to the surface and from a spot on the surface to spreading bands.

2. High tensile stresses are triggered across the sheet thickness, especially in the interior .-zone away from the surface plane. This triaxiality condition stimulates further voiding and simultaneously induces localized necking. This explains the acceleration of voiding demonstrated in Fig. 6.

3. The width of the band at bifurcation must be a material constant. Since the critical stresses vary so little with a large variation of $h / L\left(10^{2}-10^{4}\right)$, listed in Table 1, we are not able to predict a band width of microstructural scale by purely continuum mechanics. The non-local sense of employing a characteristic length suggested by BAZANT [1988] is preferable.

4. On the one hand, the rate of porosity development is a controlling factor for favouring shear-band bifurcation; on the other hand, bifurcation promotes further voiding and localization. The strong interaction between void damage and shear banding is the striking feature in material bifurcation. 
Acknowledgements - The support of this work by the National Natural Science Foundation of China and the Chinese Academy of Sciences is gratefully acknowledged.

\section{REFERENCES}

1959 HrLl, R. "Some Basic Principles in the Mechanics of Solids without a Natural Time," J. Mech. Phys. Solids, 7, 209.

1967 HILl, R., "Eigenmodal Deformations in Elastic/Plastic Continua," J. Mech. Phys. Solids, 15, 371.

1975 Hill, R., and Hutchinson, J.W., "Bifurcation Phenomena in the Plane Tension Test," J. Mech. Phys. Solids, 23, 239.

1975 McMreking, R.M., and Rice, J.R., "Finite Element Formulations for Problems of Large ElasticPlastic Deformation,” Int. J. Solids Structures, 11, 601.

1977 Gurson, A.L., "Continuum Theory of Ductile Rupture by Void Nucleation and Growth: Part I Yield Criteria and Flow Rules for Porous Ductile Media," J. Engng Mater. Tech., 99, 2.

1978 Yamamoto, H., "Conditions for Shear Localization in the Ductile Fracture of Void Containing Materials," Int. J. Fracture, 14, 347.

1979 Burke, M.A., and Nix, W.D., "A Numerical Study of Seeking in the Plane Tension Test," Int. J. Solids Structures, 15, 379.

1980 ANAND, L., and SPITzIG, W.A., "Initiation of Localized Shear Bands in Plane Strain," J. Mech. Phys. Solids, 28, 113.

1983 LI, G.C., and Howard, I.C., "The Effect of Strain-Softening in the Matrix Material during Void Growth," J. Mech. Phys. Solids, 31, 85.

1984 OHNo, N., and Hutchinson, J.W., "Plastic Flow Localization due to Non-Uniform Void Distribution," J. Mech. Phys. Solids, 32, 63.

1987 TvergaARD, V., "Effect of Yield Surface Curvature and Void Nucleation on Plastic Flow Localization," J. Mech. Phys. Solids, 35, 43.

1988 BAZANT, Z.P., "Softening Instability: Part I - Localization into a Planar Band," Trans. ASME, J. Appl. Mech., 55, 517.

1988 Becker R., Needleman, A., Richmond, O., and Tvergaard, V., "Void Growth and Failure in Notched Bars," J. Mech. Phys. Solids, 36, 317.

1988 LI, G.C., "The Mechanical Condition of Shear-Band Bifurcation," Acta Mech. Sinica (English edn), $4,363$.

1989 Li, G.C., Guennouni, T., and Francois, D., "Influence of Secondary Void Damage in the Matrix Material around Voids," Fatigue Fract. Engng Mater. Struct., 12, 105.

1990 LI, G.C., "Numerical Analysis of Shear-Band Bifurcation," Acta Mech. Sinica (English edn), 6, 22.

1990 Mear, M.E., "On the Plastic Yielding of Porous Metals," Mech. Mater., 9, 33.

1990 SchreYer, H.L., "Analytical Solutions for Nonlinear Strain-Gradient Softening and Localization," Trans. ASME, J. Appl. Mech., 57, 522.

1991 LI, G.C., "Strain Softening in Constitutive Behaviour and Material Bifurcation," in WANG R., and DruCker, D.C. (eds.), IUTAM Symposium Beijing/China 1991, Constitutive Relations for Finite Deformation of Polycrystalline Metals, Springer-Verlag, Berlin, pp. 303-17.

1991 Muhlhaus, H.B. and AIfantis, E.C., "A Variational Principle for Gradient Plasticity," Int. J. Solids Structures, 28, 845.

1992 LEE, Y.K., "Shear Banding and Material Instability in Finitely Deformed Compressible Materials: An Example in Thin Sheets," Int. J. Plasticity, 8, 543.

1992 LI, G.C., LIU, H.Q, Du, M.L., Hong, Y.S, and ZHANG, X., "Crack Tip Behaviour and Crack Propagation in Ductile Materials," Fatigue Fract. Engng Mater. Struct., 15, 187.

1992 LIU, H.Q., and LI, G.C., "Plastic Stress-Strain Fields and Blunting Effects during Large Deformations," Engng Fract. Mech., 42, 737.

16 Division

Institute of Mechanics

15, Zhong Guan Cun

Beijing, 100080, People's Republic of China

(Received in final revised form 28 September 1994) 